\title{
Molecular Dynamics Simulations of Ligand Dissociation from Thyroid Hormone \\ Receptors: Evidence of the Likeliest Escape Pathway and its Implications for the \\ Design of Novel Ligand
}

Leandro Martínez, Paul Webb, Igor Polikarpov and Munir S. Skaf.

\section{Supporting Information}

\section{Contents:}

Molecular Dynamics Simulations Details $\quad$ S1

Integrated forces for all individual simulations $\quad$ S5

CHARMM topology files for TR ligands $\quad$ S6

$\begin{array}{ll}\text { CHARMM parameter files for TR ligands } & \text { S13 }\end{array}$

$\begin{array}{ll}\text { References of this material } & \text { S15 }\end{array}$

\section{Molecular Dynamics Simulations Details}

Coordinates for LBD structures used in the simulations were obtained from: Prof.

Robert Fletterick's home-page ${ }^{1,2}$ for $\mathrm{TR} \alpha 1$ bound to $\mathrm{T} 3^{1,2}$ refined to $2.0 \AA$ resolution; the Protein Data Bank for structures of TR $\alpha 1$ bound $\mathrm{IH} 5^{3}$ and TR $\beta$ bound to $\mathrm{IH} 5^{3}$ and $\mathrm{GC} 24^{4}$ (PDB ids and resolutions: TR $\alpha 1$-IH5: 1NAV, $2.5 \AA$; TR $\beta-I H 5: 1 N A X, 2.7 \AA$; TR $\beta-G C 24:$ 1Q4X, $2.8 \AA$ ). Structures for TR $\alpha 1$ and TR $\beta$ bound to GC1 and Triac, and TR $\beta$ bound to T3 were obtained from Polikarpov and co-workers (unpublished) and are refined to $1.85 \AA$, $2.1 \AA, 2.6 \AA, 2.4 \AA$ and $2.6 \AA$ resolutions, respectively ${ }^{5}$. Missing residues were modeled, 
particularly the $\Omega$-loop in TR $\beta$ structures. The modeling was performed by finding the lowest energy structure that containing the missing residues for which the $\mathrm{N}$-terminal and C-terminal ends fit the restraints of the positions they had to have in order to be well incorporated into the overall LBD structure. The complete structures were then solvated with the package Packmol $^{6}$ by a water shell of at least $15 \AA$ (16500 water molecules) around the LBD with sodium and chloride ions added in order to make the overall systems electrically neutral. No periodic boundary conditions were used. These solvated systems were minimized by 1000 steps of conjugate-gradient minimization as implemented in $\mathrm{NAMD}^{7}$, keeping all the protein and ligand atoms, except the modeled ones, fixed. Then, 100 ps of thermalization at $298.15 \mathrm{~K}$ with velocity scaling at every 1 ps was performed, again keeping all atoms, except modeled ones, fixed. Following these first steps of thermalization, another 100 ps of simulations were performed with velocity scaling at every $1 \mathrm{ps}$, but keeping only the $\alpha$-carbon atoms of non-modeled residues fixed. Finally, 100 ps of thermalization with velocity scaling at 1 ps were performed without any position restraint. The SMD simulations were performed from the final structures obtained from these last simulations, lasted $1 \mathrm{~ns}$, and were performed without velocity scaling. We also performed 1 ns MD simulations without steering for all systems and no dissociation was observed, as expected. CHARMM parameters were used. The parameters for the ligands were obtained by group analogy from the CHARMM set. All atoms were treated explicitly. The charges of the ligands were obtained from ab-initio calculations as described in our previous work ${ }^{8}$. All parameters are available bellow. Electrostatic interactions were cutoff at $14 \AA$ with a switching function starting at $12 \AA$. All simulations were performed with NAMD ${ }^{7}$. A timestep of 2 fs was used. Energy conservation was always better than $0.5 \%$ in all simulations. The steering velocity was $0.032 \AA / \mathrm{ps}$ and the force constant used was $4.00 \mathrm{kcal} \mathrm{mol}^{-1} \AA^{-2}$. 
This velocity is chosen to promote dissociation in the simulation time desired and the force constant is chosen such that it is sufficiently small to render fluctuations of the applied force, but also large enough so that the contraction of the harmonic spring due to the advancement of the ligand by 1 or $2 \AA$ results in a noticeable drop in the force ${ }^{9}$. The pulling directions were chosen from the results presented in our previous work ${ }^{8}$. At least three SMD simulations were performed for each system, each with a direction corresponding to the paths previously found. ${ }^{8}$ A total of 35 SMD simulations were performed. The force profiles shown in Figure 3 are running averages for clarity. All the parameters used are available bellow in NAMD/CHARMM topology and parameter formats. Some simulations were repeated with variations in the pulling direction to confirm the results.

The external force was applied as follows:

For each path, the pulling direction was chosen as the direction of the vector connecting the pulled atom to the average position between two $\mathrm{C} \alpha$ atoms belonging to the protein, as shown in the figure on the right.

For each simulation slightly different pulling directions were obtained in this way for each path, providing some sampling of the pulling
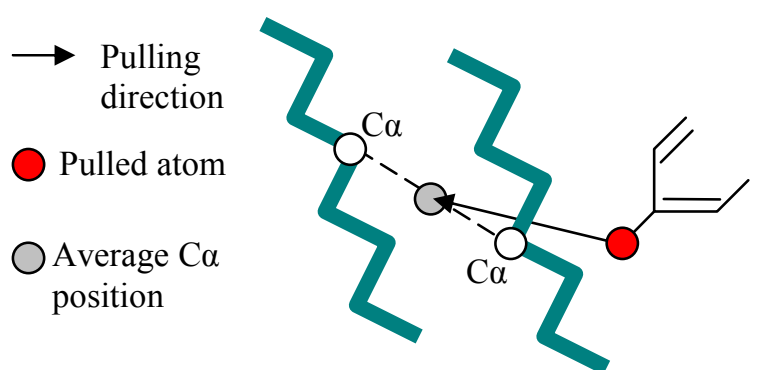
directions. The $\mathrm{C} \alpha$ atoms were chosen in order that the exit route was similar to the ones observed in our previous LES simulations. ${ }^{8}$ Therefore, for each path the pulling direction is specified by the pulled atom and the average position of two $\mathrm{C} \alpha$ atoms chosen from a residue range in each side of the escape cavity.

Path I. Pulled atom: Phenolic oxygen. Direction: the average position of one $\mathrm{C} \alpha$ atom in the top part of the loop connecting H11 and H12 or the bottom part of H12 (the Ca 
atom belongs to one of the residues in the range: L396-P398, considering TR $\alpha$ numbering) and the other belonging to the bottom part of $\mathrm{H} 3$ (residue range in TR $\alpha$ : E213-K220).

Path II. Pulled atom: Carbon of the phenolic ring directly bound to the phenolic oxygen. Direction: Average position of one $\mathrm{C} \alpha$ atom in the bottom part of $\mathrm{H} 8$ (residue range in TR $\alpha$ : G291-V295) and one C $\alpha$ atom in the bottom of H11 (residue range in TR $\alpha$ : H381-H387)

Path III. Pulled atom: Carbon atom of the carboxilate. Direction: Average position of one $\mathrm{C} \alpha$ atom from the middle of $\mathrm{H} 3$ (residue range in TR $\alpha$ : I221-A225) and one $\mathrm{C} \alpha$ atom from the $\beta$-hairpin or the loop between H1 and H2 (residues in TR $\alpha$ : S277-S279 or A180). 
Supplementary Table 1. Integrated forces computed from all the simulations in pN.ns. Asterisks indicate that dissociation was not observed. If more than one simulation was performed (by varying the pulling direction), both results are indicated separated by a bar.

\begin{tabular}{cccc}
\hline System & Path I & Path II & Path III \\
\hline TR $\alpha-$ T3 & $421^{*} / 559^{*}$ & $689^{*}$ & 332 \\
TR $\beta-$ T3 & 502 & $754^{*}$ & 355 \\
TR $\alpha$-Triac & $722^{*}$ & $610^{*} / 628^{*}$ & 413 \\
TR $\beta-$ Triac & $612^{*} / 577^{*}$ & $547^{*}$ & 385 \\
TR $\alpha-$ IH5 & 539 & $697^{*}$ & 325 \\
TR $\beta-$ IH5 & 485 & 504 & 308 \\
TR $\alpha-G C 1$ & $677^{*} / 664^{*}$ & $657^{*}$ & 355 \\
TR $\alpha-G C 1{ }^{\text {a }}$ & $636^{*} / 535^{*}$ & $585^{*}$ & 348 \\
TR $\beta-G C 1$ & 556 & $653^{*}$ & 340 \\
TR $\beta-G C 24$ & $601 *$ & $650^{*}$ & 562 \\
\hline
\end{tabular}




\section{Topology files}

* CHARMM topology file for thyroid hormone and thyroid hormone

* analogs

*

271

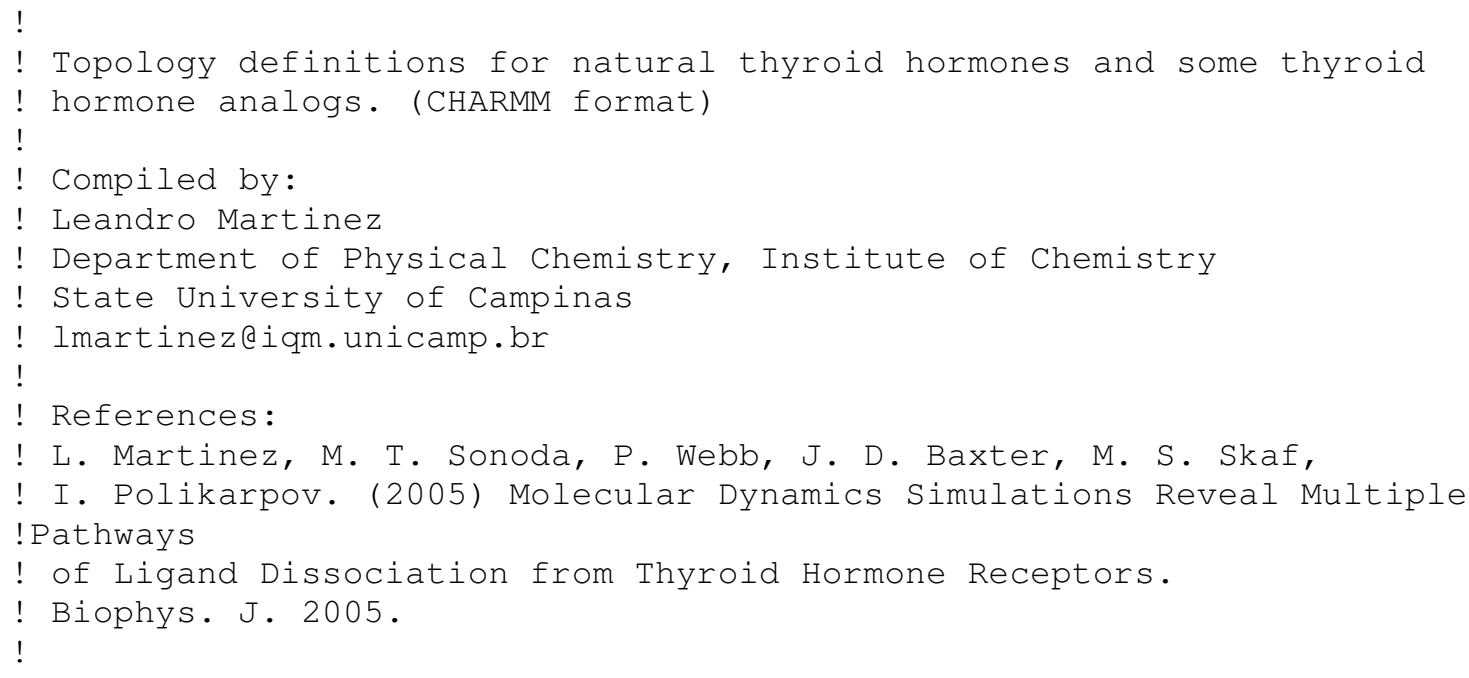

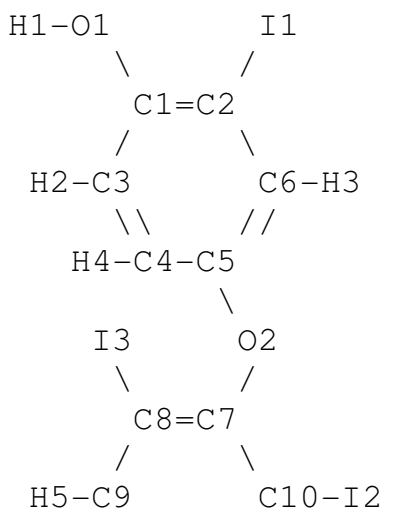




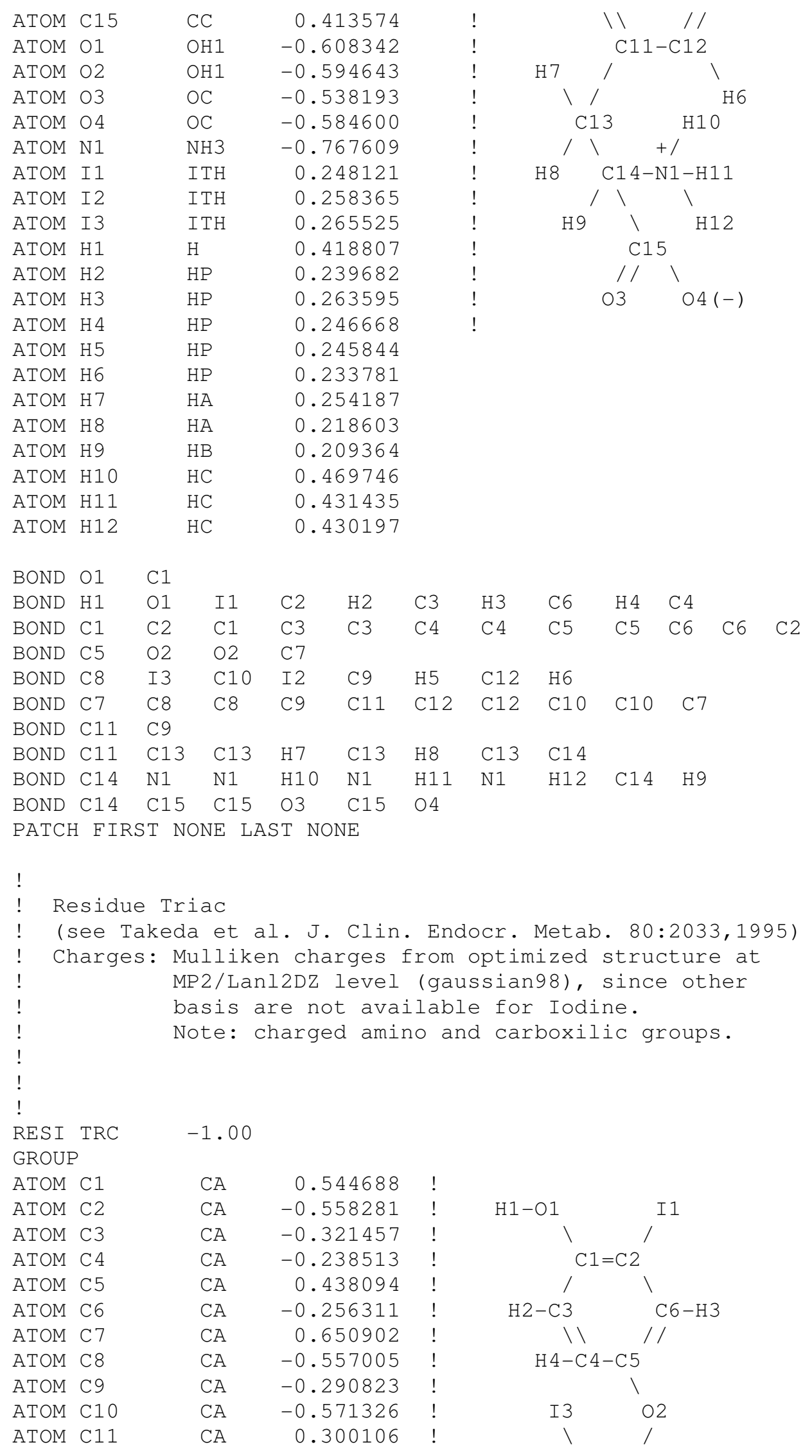









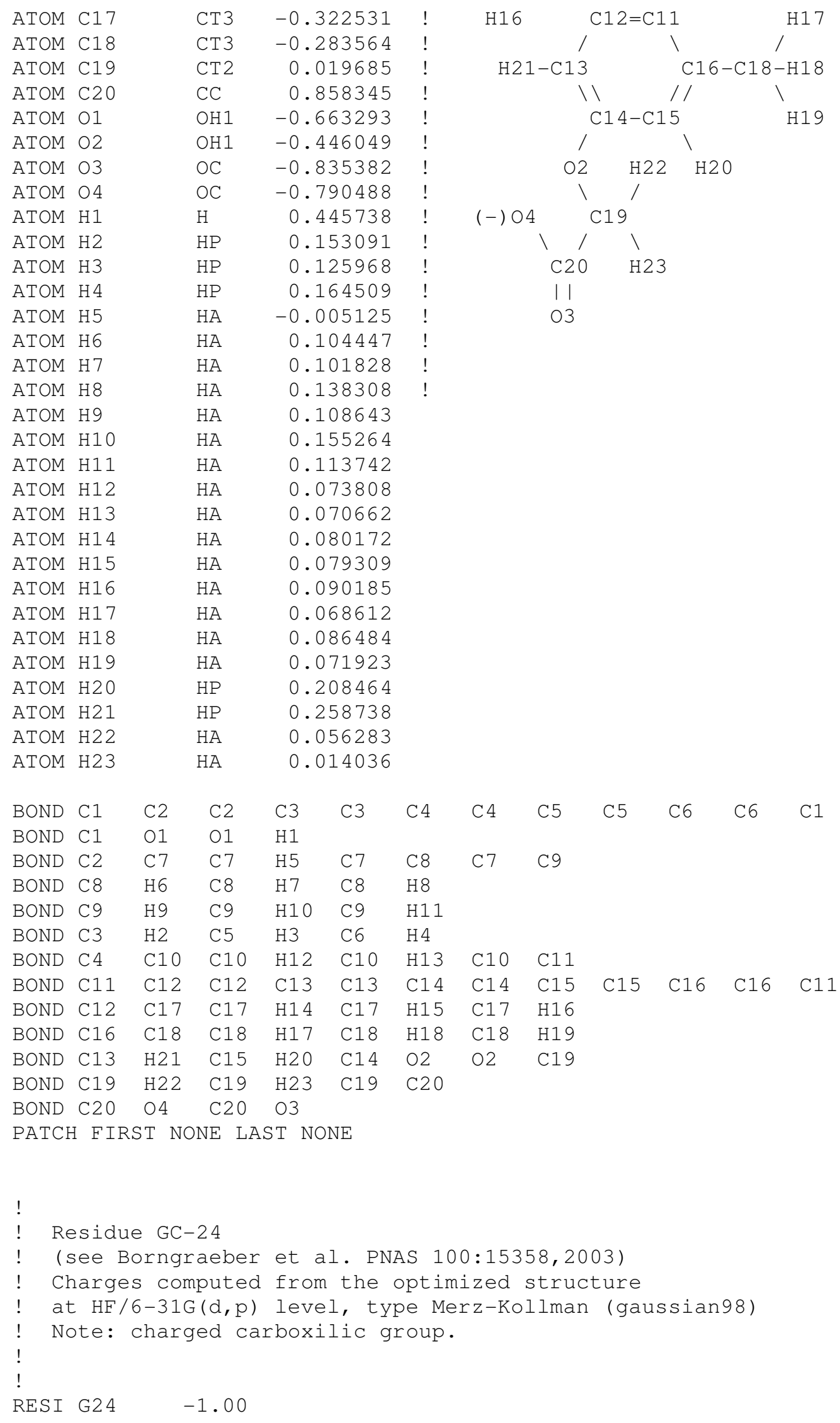




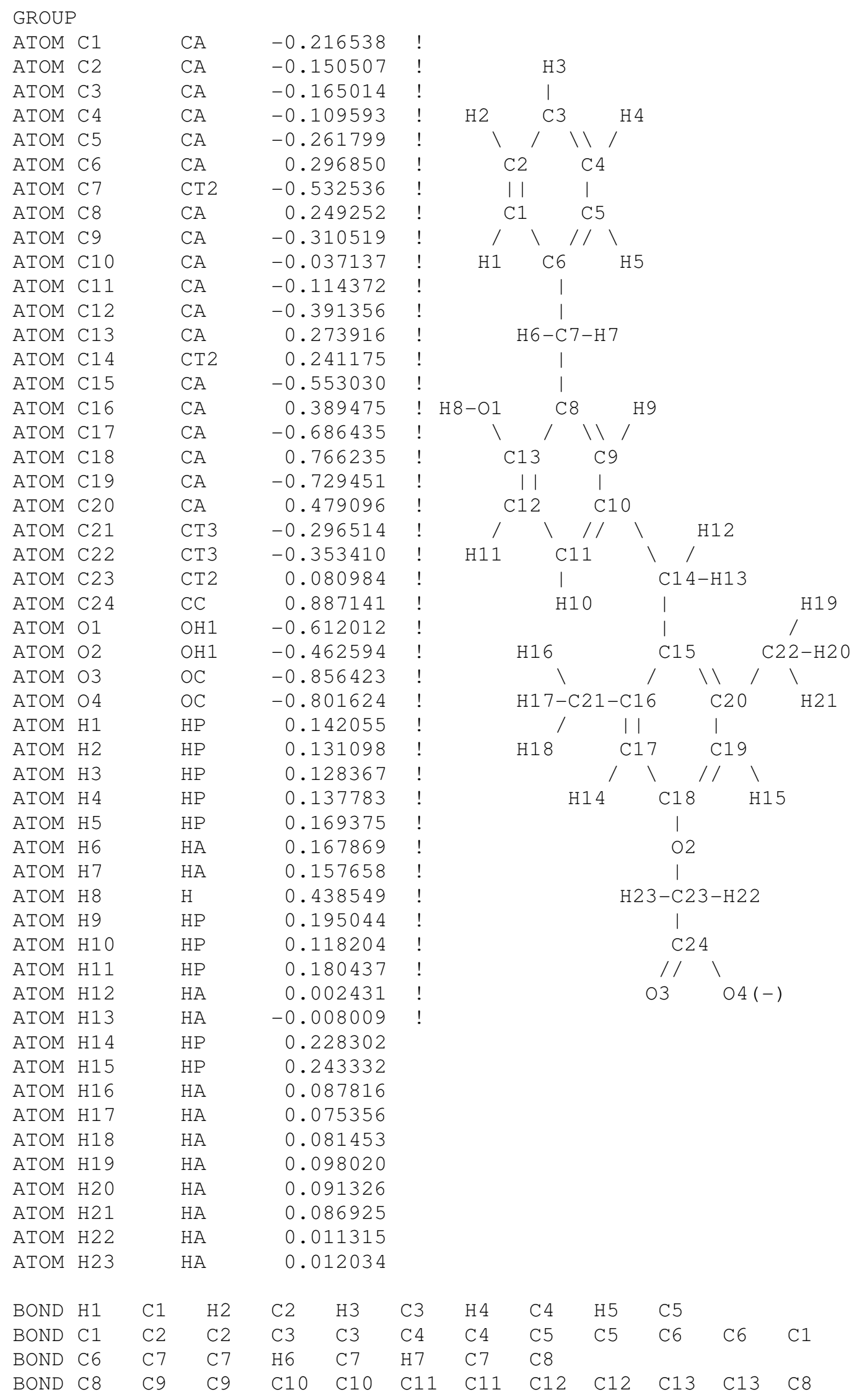




\begin{tabular}{|c|c|c|c|c|c|c|c|c|c|c|c|c|}
\hline BOND & C9 & H9 & C11 & $\mathrm{H} 10$ & C12 & $\mathrm{H} 11$ & C13 & 01 & 01 & H8 & & \\
\hline BOND & $\mathrm{C} 10$ & C14 & C14 & $\mathrm{H} 12$ & $\mathrm{C} 14$ & $\mathrm{H} 13$ & C14 & C15 & & & & \\
\hline BOND & C15 & C16 & C16 & C1 7 & C1 7 & C18 & C18 & C19 & C19 & C20 & $\mathrm{C} 20$ & C15 \\
\hline BOND & C1 7 & $\mathrm{H} 14$ & C19 & H1 5 & & & & & & & & \\
\hline BOND & C16 & C21 & C21 & $\mathrm{H} 16$ & C21 & H1 7 & C21 & H1 8 & & & & \\
\hline BOND & $\mathrm{C} 20$ & C22 & C22 & H19 & C22 & H2O & C22 & H 21 & & & & \\
\hline BOND & C18 & 02 & O2 & C23 & C23 & H23 & C23 & H 22 & & & & \\
\hline BOND & C23 & C24 & C2 4 & 03 & C2 4 & 04 & & & & & & \\
\hline
\end{tabular}

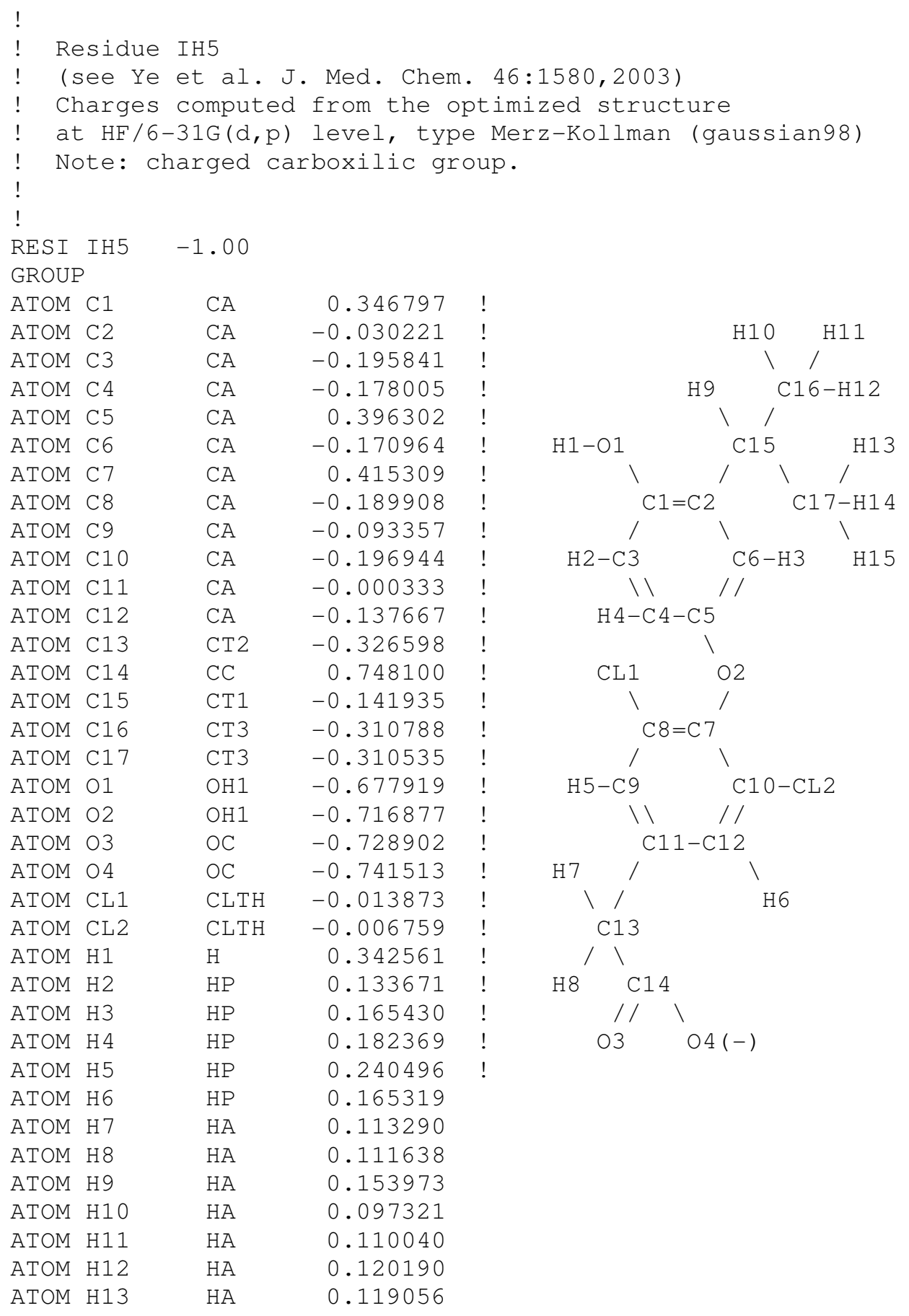




\begin{tabular}{|c|c|c|c|c|c|c|c|c|c|c|c|c|}
\hline ATOM & $\mathrm{H} 14$ & & & 0. & 97408 & & & & & & & \\
\hline ATOM & H1 5 & & & 0.1 & 09669 & & & & & & & \\
\hline BOND & $\mathrm{C} 1$ & $\mathrm{C} 2$ & $\mathrm{C} 2$ & C6 & $\mathrm{C} 6$ & C5 & C5 & C 4 & C4 & C3 & C3 & $\mathrm{C} 1$ \\
\hline BOND & $\mathrm{C} 1$ & 01 & 01 & $\mathrm{H} 1$ & & & & & & & & \\
\hline BOND & $\mathrm{C} 2$ & C15 & C15 & H9 & & & & & & & & \\
\hline BOND & C15 & C16 & C16 & $\mathrm{H} 10$ & C16 & H11 & C16 & $\mathrm{H} 12$ & & & & \\
\hline BOND & C15 & C1 7 & C1 7 & H13 & C1 7 & H1 4 & C1 7 & H1 5 & & & & \\
\hline BOND & C3 & $\mathrm{H} 2$ & C4 & H4 & C6 & H3 & & & & & & \\
\hline BOND & $\mathrm{C} 5$ & $\mathrm{O} 2$ & $\mathrm{O} 2$ & C 7 & & & & & & & & \\
\hline BOND & C 7 & C 8 & $\mathrm{C} 8$ & $\mathrm{C9}$ & C9 & C11 & C11 & $\mathrm{C} 12$ & $\mathrm{C} 12$ & C10 & $\mathrm{C} 10$ & C 7 \\
\hline BOND & C8 & CL1 & C9 & H5 & C12 & H6 & C10 & CL2 & & & & \\
\hline BOND & C11 & C13 & C13 & H 7 & C13 & H8 & C13 & C14 & & & & \\
\hline BOND & C14 & 03 & C14 & 04 & & & & & & & & \\
\hline
\end{tabular}




\section{Parameters:}

* CHARMM parameter file for thyroid and thyroid hormone

* analogs

$\star$

BONDS

!

$\mathrm{IV}(\mathrm{bond})=\mathrm{Kb}(\mathrm{b}-\mathrm{b} 0) * \star 2$

!

! Kb: $\mathrm{kcal} / \mathrm{mole} / \mathrm{A} * \star 2$

! bo: A

$!$

! atom type $\mathrm{Kb} \quad$ bo

!

ITH CA $166.0 \quad 2.080$ ! Iodine bond in T3

CLTH CA 193.0 1.745 ! From JMC 46,24,2003

CA CT1 230.0 1.490 ! Identical to CA CT2 and CA CT3

ANGLES

!

! V (angle $)=$ Ktheta (Theta - Theta 0$) * * 2$

!

!V (Urey-Bradley) $=\operatorname{Kub}(\mathrm{S}-\mathrm{S} 0) * \star 2 !$

! Ktheta: $\mathrm{kcal} / \mathrm{mole} / \mathrm{rad} * * 2$

! Theta0: degrees

!Kub: $\mathrm{kcal} / \mathrm{mole} / \mathrm{A} * \star 2$ (Urey-Bradley)

! SO: A

!

!atom types Ktheta Thetao Kub so

!

!

$\begin{array}{lllllllll}\text { ITH } & \text { CA } & \text { CA } & 45.8 & 120.0 & 0.0 & 0.000 & \text { ! From CT3 CA CA } \\ \text { CA } & \text { OH1 } & \text { CA } & 70.0 & 123.8 & 0.0 & 0.000 & \text { ! C-O-C ether } \\ \text { CA } & \text { CA } & \text { CLTH } & 70.0 & 120.0 & 0.0 & 0.000 & \text { ! From JMC } 46,24,2003 \\ \text { CA } & \text { CA } & \text { CT1 } & 45.8 & 122.3 & 0.0 & 0.000 & \text { ! Id. to CA CA CT2/CT3 } \\ \text { CA } & \text { CT1 } & \text { CT3 } & 51.8 & 107.5 & 0.0 & 0.000 \text { ! Id. to CA CT1 CT2 } \\ \text { CA } & \text { CT1 } & \text { HA } & 49.3 & 107.5 & 0.0 & 0.000 & \text { ! Id. to HA CT2 CA } \\ \text { CA } & \text { CT2 } & \text { CC } & 51.8 & 107.5 & 0.0 & 0.000 \text { ! Id. to CT1 CT2 CA } \\ \text { CA } & \text { CT2 } & \text { CA } & 52.0 & 116.6 & 0.0 & 0.000 \text { ! Theta0 from ab-initio } \\ \text { CA } & \text { OH1 } & \text { CT2 } & 70.0 & 121.3 & 0.0 & 0.000 \text { ! Theta0 from ab-initio } \\ \text { CC } & \text { CT2 } & \text { OH1 } & 52.0 & 117.9 & 0.0 & 0.000 \text { ! Theta0 from ab-initio }\end{array}$

DIHEDRALS

!

$! \mathrm{V}($ dihedral $)=\operatorname{Kchi}(1+\cos (\mathrm{n}(\operatorname{ch} i)-\operatorname{delta}))$

!

!Kchi: $\mathrm{kcal} / \mathrm{mole}$

!n: multiplicity

!delta: degrees

!

!atom types

!

$\begin{array}{lllllll}\mathrm{CA} & \mathrm{OH} 1 & \mathrm{CA} & \mathrm{CA} & 4.20 & 2 & 34.00 \quad \text { ! }\end{array}$

OH1 CA CA ITH $\quad 4.20 \quad 2 \quad 180.00$ !From OH1 CA CA HP

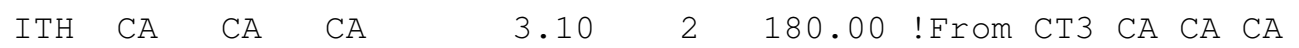




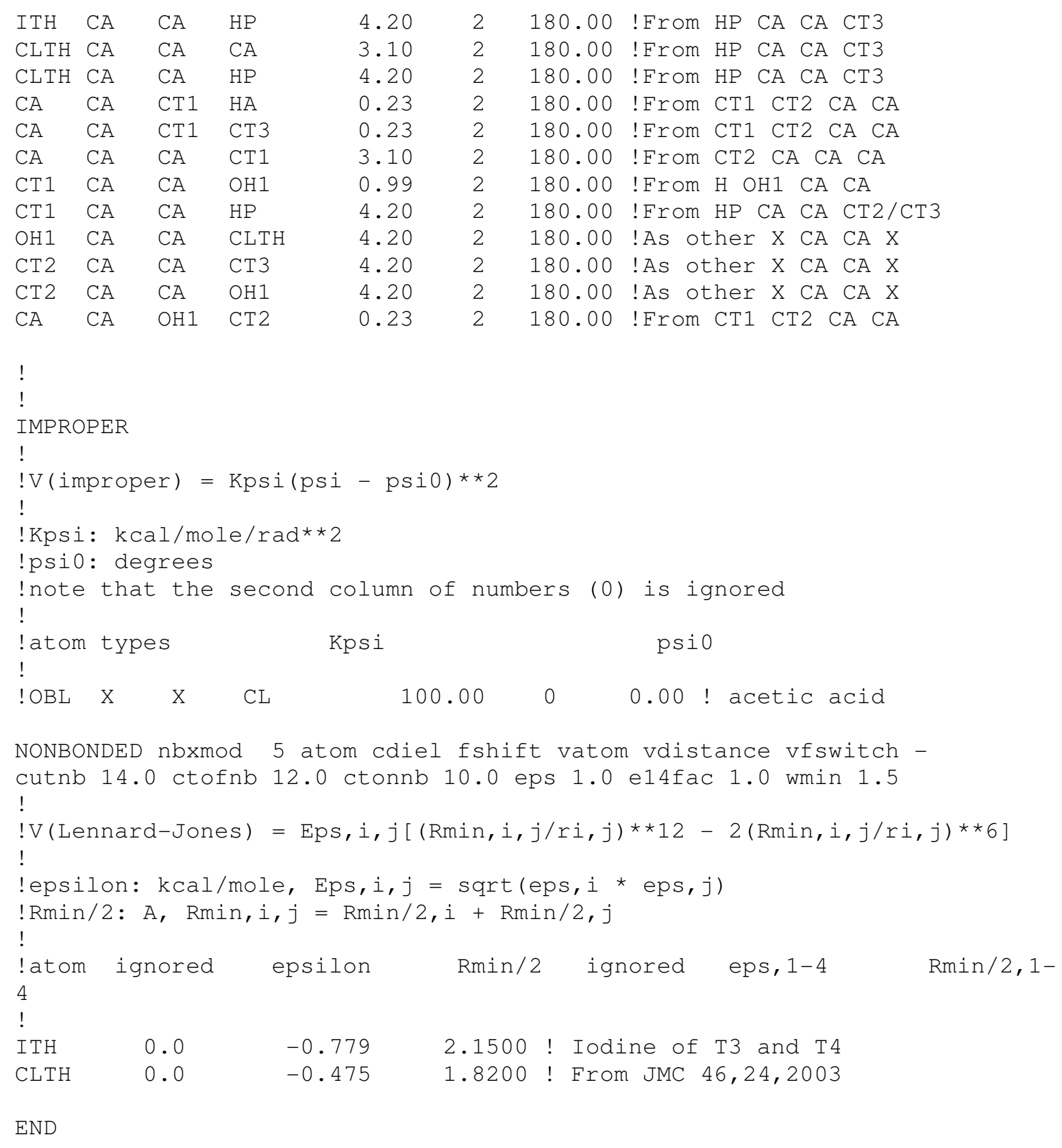




\section{References of this material}

1. http://www.msg.ucsf.edu/flett

2. Wagner, R. L.; Aprietti, J. W.; McGrath, M. E.; West, B. L.; Baxter, J. D.; Fletterick, R.

J. A structural role for hormone in the thyroid hormone receptor. Nature 1995, 378, 690697.

3. Ye, L.; et al. Thyroid Receptor Ligands. 1. Agonist Ligands Selective for the Thyroid Receptor $\beta 1$. J. Med. Chem. 2003, 46, 1580-1588.

4. Borngraeber, S.; Budny, M. J.; Chiellini, G.; Lima, S. T. C; Togashi, M.; Webb, P.; Baxter, J. D.; Scanlan, T. S.; Fletterick, R. J. Ligand selectivity by seeking hydrophobicity in thyroid hormone receptors. Proc. Natl. Acad. Sci. USA 2003, 100, 15358-15363.

5. Nunes, A. M.; et al. Crystallization and preliminary X-ray diffraction studies of isoform $\alpha 1$ of the human thyroid hormone receptor ligand-binding domain. Acta Crystallogr. D 2004, 60, 1867-1870.

6. Martínez, J. M.; Martínez, L. Packing optimization for the automated generation of complex system's initial configurations for molecular dynamics and docking. J. Comp. Chem. 2003, 24, 819-825. (http://www.ime.unicamp.br/ martinez/packmol)

7. Kalé, L.; Skeel, R.; Bhandarkar, M.; Brunner, R.; Gursoy, A.; Krawetz, N.; Phillips, J.; Shinozaki, A.; Varadarajan, K.; Schulten, K. NAMD2: Greater scalability for parallel molecular dynamics. J. Comp. Phys. 1999, 151, 283-312.

8. Martínez, L.; Sonoda, M. T.; Webb, P.; Baxter, J. D.; Skaf, M. S.; Polikarpov, I. Molecular Dynamics Simulations Reveal Multiple Pathways of Ligand Dissociation from Thyroid Hormone Receptors. Biophys. J. 2005, 89, 2011-2023.

9. Kosztin, D.; Izrailev, S.; Schulten, K. Unbinding of Retinoic Acid from its Receptor Studied by Steered Molecular Dynamics. Biophys. J. 1999, 76, 188-197 